\title{
A THEOREM OF LJUNGGREN AND JACOBSTHAL ON BERNOULLI NUMBERS
}

\author{
L. CARLITZ
}

1. Introduction. Jacobsthal [1] has recently proved the following formulas

$$
\begin{aligned}
& \sum_{r=1,(r, m)=1}^{m}(r+m B)^{2 q}=m B_{2 q} \prod_{p \mid m}\left(1-p^{2 q-1}\right), \\
& \sum_{r=1,(r, m)=1}^{[(m-1) / 2]}(r+m B)^{2 q}=\frac{1}{2} m B_{2 q} \prod_{p \mid m}\left(1-p^{2 q-1}\right) \quad(m>2),
\end{aligned}
$$

where $B_{2 q}$ denotes the Bernoulli number in the even suffix notation and the products are extended over all primes $p$ dividing $m$. Earlier Ljunggren [2] had proved such results in the special case $m=p$. In proving these results use is made of the formula

$$
\sum_{r=0}^{m-1}(r+m B)^{2 q}=m B_{2 q}
$$

ascribed to Blissard.

Now (1.3) can be thought of as a special case of the multiplication theorem for the Bernoulli polynomial [3, p. 21]

$$
\sum_{s=0}^{m-1} B_{k}\left(x+\frac{s}{m}\right)=m^{1-k} B_{k}(k x) .
$$

Since a like theorem holds for the Euler polynomial $E_{m}(x)$ as well as for the Bernoulli and Euler polynomials of higher order, this suggests formulas like (1.1) and (1.2) in those cases. Such results are derived in this note.

2. In (1.4) take $x=0$. Then

$$
\begin{aligned}
m^{1-k} B_{k}=B_{k}+\sum_{s=1}^{m-1} & B_{k}\left(\frac{s}{m}\right)=B_{k}+\sum_{d \mid m} \sum_{s=1,(s, m)=d}^{m-1} B_{k}\left(\frac{s}{m}\right) \\
& =B_{k}+\sum_{d e=m} \sum_{r=1,(r, e)=1}^{e-1} B_{k}\left(\frac{r}{e}\right) \\
& =B_{k}+\sum_{d e=m, d<m} \psi_{k}(e),
\end{aligned}
$$

Received by the editors December 6, 1952. 
where

$$
\psi_{k}(e)=\sum_{r=1,(r, e)=1}^{e-1} B_{k}\left(\frac{r}{e}\right) \quad(e>1), \psi_{k}(1)=B_{k} .
$$

Thus (2.1) becomes

$$
m^{1-k} B_{k}=\sum_{e \mid m} \psi_{k}(e)
$$

Applying the Möbius inversion formula to (2.2), we get at once

$$
\psi_{k}(m)=\phi_{1-k}(m) B_{k}
$$

where

$$
\phi_{1-k}(m)=m^{1-k} \prod_{p \mid m}\left(1-p^{k-1}\right) .
$$

It is easily verified that for $k=2 q,(2.4)$ reduces to (1.1).

In the next place we recall the formula $[3, \mathrm{p} .21]$

$$
B_{k}(1-x)=(-1)^{k} B_{k}(x) \text {. }
$$

Then by (2.2), (2.4), and (2.5) we get

$$
\sum_{r=1,(r, m)=1}^{[(m-1) / 2]} B_{2 k}\left(\frac{r}{m}\right)=\frac{1}{2} \phi_{1-2 k}(m) B_{2 k} \quad(m>2),
$$

which is equivalent to (1.2).

3. In the case of the Euler polynomials we have [3, p. 24]

$$
\begin{gathered}
m^{-k} E_{k}(m x)=\sum_{s=0}^{m-1}(-1)^{s} E_{k}\left(x+\frac{s}{m}\right) \quad(m \text { odd }), \\
E_{k}(1-x)=(-1)^{k} E_{k}(x) .
\end{gathered}
$$

Exactly as in (2.1) we have

$$
m^{-k} E_{k}(0)=\sum_{e \mid m} \chi_{k}(e)
$$

where

$$
\chi_{k}(e)=\sum_{r=1,(r, e)=1}^{e-1}(-1)^{r} E_{k}\left(\frac{r}{e}\right)
$$

and $\chi_{k}(1)=E_{k}(0)$. Applying the inversion formula to (3.3), we get

$$
\sum_{r=1,(r, e)=1}^{m}(-1)^{r} E_{k}\left(\frac{r}{m}\right)=\phi_{1-k}(m) E_{k}(0) \quad(m \text { odd }) .
$$


In view of (3.2) this implies

$$
\sum_{r=1,(r, m)=1}^{(m-1) / 2}(-1)^{r} E_{2 k-1}\left(\frac{r}{m}\right)=\frac{1}{2} \phi_{-2 k}(m) E_{2 k-1}(0) \quad(m \text { odd }>2) .
$$

Thus (3.4) and (3.5) furnish analogues of Jacobsthal's theorem.

4. Turning now to the Bernoulli polynomials of order $n$, we put

$$
B_{k}^{(n)}(x)=B_{k}^{(n)}\left(x \mid \omega_{1}, \cdots, \omega_{n}\right), B_{k}^{(n)}=B_{k}^{(n)}\left(0 \mid \omega_{1}, \cdots, \omega_{n}\right) .
$$

We require the formula $[3$, p. 135]

$$
\sum_{s_{1}, \cdots, s_{n}=0}^{m-1} B_{k}^{(n)}\left(x+\frac{S_{1} \omega_{1} \cdots+S_{n} \omega_{n}}{m}\right)=m^{n-k} B_{k}^{(n)}(m x) .
$$

As in $\$ 2$ we find that

$$
m^{n-k} B_{k}^{(n)}=B_{k}^{(n)}+\sum_{d \mid m} \sum_{\substack{s_{1}, \cdots, s_{n}=0 \\\left(s_{1}, \cdots, s_{n}, m\right)=d}}^{m-1} B_{k}^{(n)}\left(\frac{s_{1} \omega_{1}+\cdots+s_{n} \omega_{n}}{m}\right),
$$

where the combination $s_{1}=\cdots=s_{n}=0$ is omitted. Then

$$
m^{n-k} B_{k}^{(n)}=B_{k}^{(n)}
$$

$$
+\sum_{d e=m, d<m} \sum_{\substack{r_{1}, \cdots, r_{n}=1 \\\left(r_{1}, \cdots, r_{n}, e\right)=1}}^{\sigma-1} B_{k}^{(n)}\left(\frac{r_{1} \omega_{1}+\cdots+r_{n} \omega_{n}}{e}\right) .
$$

If we put

$$
\psi_{k}^{(n)}(m)=\sum_{\substack{r_{1}, \cdots, r_{n}=1 \\\left(r_{1}, \cdots, r_{n}, m\right)=1}}^{m-1} B_{k}^{(n)}\left(\frac{r_{1} \omega_{1}+\cdots+r_{n} \omega_{n}}{m}\right)(m>1)
$$

and $\psi_{\mathbf{k}}^{(n)}(1)=B_{\boldsymbol{k}}^{(n)}$, then (4.2) becomes

$$
m^{n-k} B_{k}^{(n)}=\sum_{e \mid m} \psi_{k}^{(n)}(e)
$$

Inverting (4.4), we get

$$
\psi_{k}^{(n)}(m)=\phi_{n-k}(m) B_{k}^{(n)} .
$$

(4.5) and (4.3) evidently furnish a generalization of (1.1). We omit the corresponding generalization of (1.2).

5. For the Euler polynomials of order $n$ we put 


$$
E_{k}^{(n)}(x)=E_{k}^{(n)}\left(x \mid \omega_{1}, \cdots, \omega_{n}\right),
$$

and recall the formula $[3$, p. 123$]$

$$
\begin{array}{r}
\sum_{s_{1}, \cdots, s_{n}=0}^{m-1}(-1)^{s_{1}+\cdots+s_{n}} E_{k}^{(n)}\left(x+\frac{s_{1} \omega_{1}+\cdots+s_{n} \omega_{n}}{m}\right) \\
=m^{-k} E_{k}^{(n)}(m x) \quad(m \text { odd }) .
\end{array}
$$

We now find that

$$
m^{-k} E_{k}^{(n)}(0)=\sum_{\bullet \mid m} \chi_{k}^{(n)}(e)
$$

where

$$
\chi_{k}^{(n)}(m)=\sum_{\substack{r_{1}, \cdots, r_{n}=1 \\\left(r_{1}, \cdots, r_{n}, m\right)=1}}^{m-1}(-1)^{r_{1}+\cdots+r_{n}} E_{k}^{(n)}\left(\frac{r_{1} \omega_{1}+\cdots+r_{n} \omega_{n}}{m}\right)
$$

and $\chi_{k}^{(n)}(1)=E_{k}^{(n)}(0)$. Hence (5.3) yields

$$
\chi_{k}^{(n)}(m)=\phi_{-k}(m) E_{k}^{(n)}(0)
$$

Together with (5.3), this yields a generalization of (3.4). We omit the corresponding generalizations of (3.5).

\section{REFERENCES}

1. E. Jacobsthal, Zur theories der Bernoullischen Zahlen, Det Kongelige Norske Videnskabers Selskab Forhandlinger vol. 22 (1950) pp. 107-112.

2. W. Ljunggren, Sur un theoreme de M. E. Jacobsthal, Avhandlinger Utgitt av det Norske Videnskaps-Akademi i Oslo I, Mat.-Naturv. Klasse (1947) no. 5.

3. N. E. Nörlund, Vorlesungen über Differenzenrechnung, Berlin, 1924.

DUKE UNIVERSITY 